DOI: $10.2478 / \mathrm{v} 10282-012-0005-2$

\title{
Image-guided stereotactic biopsy of infiltrative, multicentric and deep-seated supratentorial cerebral gliomas
}

\author{
F.M. Brehar ${ }^{1}$, R.M. Gorgan ${ }^{1}$, M. Lisievici ${ }^{2}$ \\ 1"Carol Davila" University of Medicine and Pharmacy Bucharest, Faculty of \\ Medicine, Department of Neurosurgery \\ 2"Bagdasar Arseni” Emergency Clinic Hospital, Department of Pathology \\ Bucharest, Romania
}

\begin{abstract}
Infiltrative cerebral gliomas remain a neurosurgical challenge despite the latest achievements in neuroimaging techniques and microsurgical approaches. In this paper the authors present their experience in 85 cases of stereotactic biopsies performed for infiltrative, multicentric and deep-seated cerebral gliomas. The stereotactic and neuroimagistic tools used for these procedures included the Leksell stereotactic system and the software: Stereotactic Planning System (SPS), NTPS 8.2. The histopatological results (according to World Health Organization (WHO) classification) were: 51 cases of glioblastomas (grade IV) $(60 \%), 7$ cases of anaplastic astrocytomas (grade III) $(8,2 \%), 13$ cases of grade II diffuse astrocytomas (15,3\%), 6 cases of grade I astrocytomas $(7,1 \%)$, one case of grade II oligodendroglioma (1,2\%), 3 cases of anaplastic oligodendrogliomas (grade III) $(3,5 \%)$, one case of grade I ganglioglioma $(1,2 \%)$, one case of anaplastic ganglioglioma (grade III) (1,2\%), and 2 cases of anaplastic ependymomas (grade III) (2,3\%). In 21 cases $(24,7 \%)$ the immunohistochemistry has been performed in order to obtain an accurate histopathological result. In this series, the early postoperative mortality was
\end{abstract}

$0 \%$, with no cases of clinically significant hemorrhages after biopsy procedures. Temporary increase of neurological deficits has been noticed in 7 patients $(8,2 \%)$. In conclusion, image-guided stereotactic biopsy represents now a safe and accurate diagnostic method for cerebral gliomas, which can favorably influence the therapeutic management of the patients.

Key words: Stereotactic biopsy, gliomas, histopathology, immunohistochemistry.

\section{Introduction}

Primary brain tumors represent a major health problem. Their incidence is approximately 10 new cases at 100.000 inhabitants per year, but the frequency has been continuously increasing during the last decades (3). Within this pathology, there is an important group represented by cerebral gliomas, which ranks first as frequency and severity of the clinical outcome. According to WHO classification of tumors, cerebral gliomas can be divided in four grades (20). Excepting grade I gliomas, which can be often cured by surgical resection, grades II to IV are virtually impossible to be cured with current therapies. Glioblastoma (grade IV) is the most frequent and malignant glial tumor and it is associated with a dismal 
prognosis expressed by a limited survival expectancy of the patients (between 6 and 12 months) (16). Anaplastic astrocytoma (grade III) has also a rapid evolution to a fatal outcome with a survival period of approximately 2-3 years (2). Grade II gliomas have a slower growth and a better outcome, but they affect younger patients and have a progressive evolution to higher degrees of malignancy (grade III-IV) (8). One of the main features of grade II, III and IV gliomas is the invasion of surrounding brain tissue, which precludes a gross-total surgical resection (5). Therefore, stereotactic biopsy, a minimal invasive surgical procedure, is indicated in many cases of gliomas, especially those which are multicentric, have an infiltrative growth pattern or are located in eloquent or surgical inaccessible areas of the brain. In this paper the authors describe their experience in 85 consecutive cases of infiltrative, multicentric and deep-seated gliomas which underwent stereotactic biopsy in our clinic.

\section{Material and method}

Eighty-five consecutive patients with infiltrative, multicentric and deep-seated supratentorial gliomas have been admitted in our clinic, between November 2008 and March 2012. All patients underwent the stereotactic biopsy according to the standard protocols. The anticoagulant or antiplatelet medication, if present, had been stopped at least 7 days before the surgical procedure. Any abnormalities in the coagulogram or platelet count were rule out before biopsy. The corticosteroid therapy had been initiated before the surgical procedure in the majority of the patients. The stereotactic and neuroimagistic tools used for these procedures included the Leksell stereotactic system and the latest software: Stereotactic Planning System
(SPS), NTPS 8.2. For application of the coordinate frame, the local anesthetics lidocaine 1\% (approximately 1,5-2 $\mathrm{ml}$ per each pin site) has been used. For anxious patients and children, we administrated mild sedatives. In three cases (one small child and two confused adult patients) we used general anesthesia. In the majority of cases (60 cases - 70,6\%) the MRI 1,5 Tesla (Philips Integra) was used for scanning the fiducial system. In the rest of the cases, including the patients under general anesthesia, we used the CT scan (Philips, Brilliance). In several cases, we made the image fusion with the MR images previously acquired. Therefore, in the majority of the cases the planning was performed on MR images: gadoliniumenhanced T1-weighted, $1 \mathrm{~mm}$ slice thickness and T2-weighted, $2 \mathrm{~mm}$ slice thickness. During the planning procedure, we chose the target within the centre of homogenously enhancing lesion, or within the gadolinium enhanced periphery of the ring enhancing lesion. If the lesions did not enhanced, we chose the target within the maximum area of hypo-intense T1weighted signal and hyper-intense T2weighted signal, respectively. When CT scan was employed, the planning was performed on enhanced 1,5 $\mathrm{mm}$ slice thickness CT images. The shortest trajectories, which avoid eloquent areas and vessels, were chosen. For operatory procedure, we usually employed general anesthesia, for patient comfort. Between 4 and 8 millimeter size tumor samples per patient were obtained using the side-cutting Sedan I biopsy needle, which were sent to the histopathological department. In several cases, there were minor intraoperative hemorrhages at the biopsy site. In all those cases we did not redraw the needle; instead we irrigated with saline solution for several minutes until the hemorrhage stopped. All 
patients underwent postoperative CT scanning. The patients had received corticotherapy, prophylactic antibiotics and prophylactic anticonvulsivant therapy until they were discharged from our department.

\section{Results}

The median age of the patients was 51 years (ranged between 7 and 75 years) and the cohort included 7 children $(8,2 \%)$ and 78 adults $(91,8 \%)$. There were 44 women $(51,8 \%)$ and 41 men $(48,2 \%)$ in our series. The age distribution in the population cohort was: 1 - 10 years: 4 cases (4,7\%); 11 20 years: 7 cases $(8,2 \%) ; 21$ - 30 years: 3 cases $(3,5 \%) ; 31$ - 40 years: 14 cases (16,5\%); 41 - 50 years: 12 cases $(14,1 \%) ; 51$
- 60 year: 23 cases $(27,1 \%), 61-70$ years: 19 cases $(22,4 \%)$ and $71-80$ years: 3 cases $(3,5 \%)$.

There were 73 cases $(85,9 \%)$ of unifocal tumors, 10 cases of multicentric tumors $(11,8 \%)$ and two cases of gliomatosis cerebri $(2,3 \%)$. The main location of unifocal tumors were in thalamus in 14 cases $(16,5 \%)$, right cerebral peduncle in one case $(1,2 \%)$, posterior third ventricle in one case $(1,2 \%)$, in basal ganglia and insula in 16 cases $(18,8 \%)$. Seventeen cases were restricted to the white matter of cerebral hemispheres $(20 \%)$. In 20 cases, there was a significant invasion in corpus callosum $(23,5 \%)$. Four cases had the typical aspect of butterfly glioblastomas (4,7\%) (Figure 1).
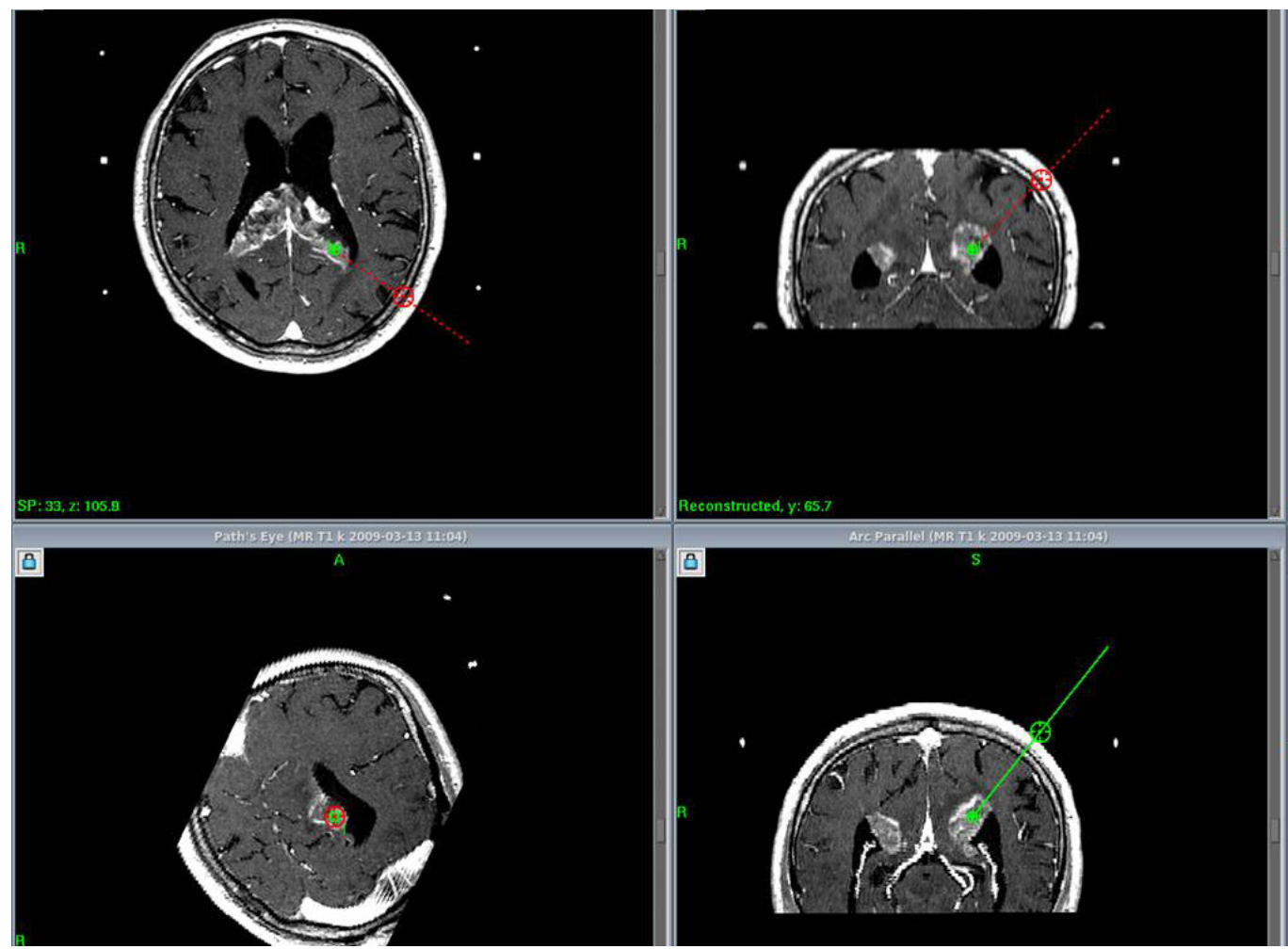

Figure 1 MR gadolinium-enhance T1-weighted images of a glioblastoma involving the splenium of the corpus callosum ("butterfly" glioblastoma) 
The histopatological results (according to World Health Organization (WHO) classification) were: 51 cases of glioblastomas (grade IV) $(60 \%), 7$ cases of anaplastic astrocytomas (grade III) $(8,2 \%)$, 13 cases of grade II diffuse astrocytomas $(15,3 \%), 6$ cases of grade I astrocytomas $(7,1 \%)$, one case of grade II oligodendroglioma $(1,2 \%), 3$ cases of anaplastic oligodendrogliomas (grade III) $(3,5 \%)$, one case of grade I ganglioglioma $(1,2 \%)$, one case of anaplastic ganglioglioma (grade III) $(1,2 \%)$, and 2 cases of anaplastic ependymomas (grade III) $(2,3 \%)$. In four cases the initial results were inconclusive
$(4,7 \%)$. Of these four cases, after a histopathological reexamination (including the immunohistochemistry techniques), two cases $(2,3 \%)$ have been interpreted as grade II fibrillary astrocytomas, one case $(1,2 \%)$ as grade I pilocytic astrocytoma and one case $(1,2 \%)$ as grade I ganglioglioma. In 21 cases (24,7\%) the immunohistochemistry was performed in order to obtain an accurate histopathological result.

Two cases of difficulty to approach, deep located gliomas (one case in the posterior part of third ventricle and one case in thalamus) are illustrated in figures 2-5.

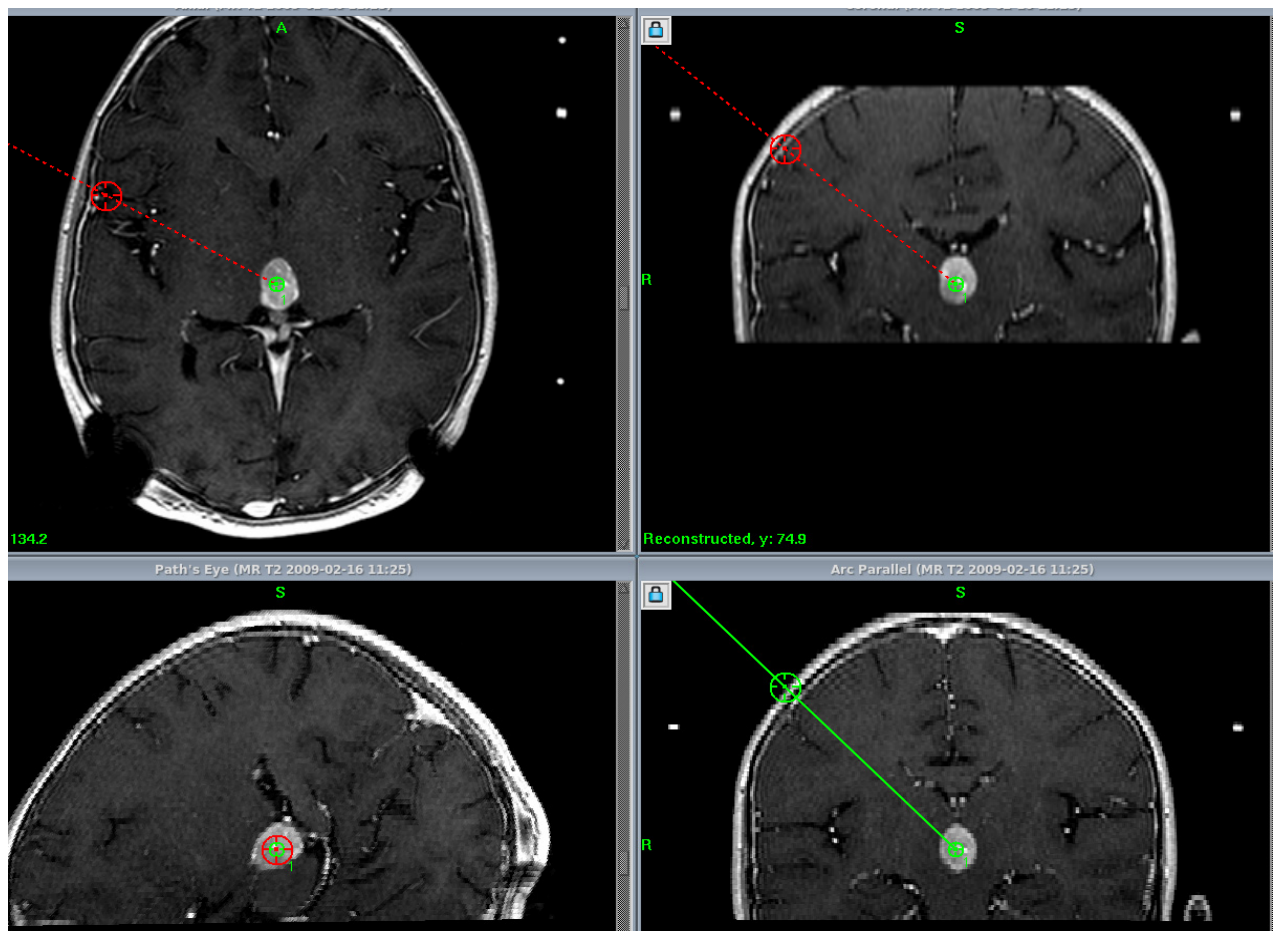

Figure 211 year-old girl with posterior third ventricle tumor mass - gadolinium-enhanced T1-weighted MR planning images 


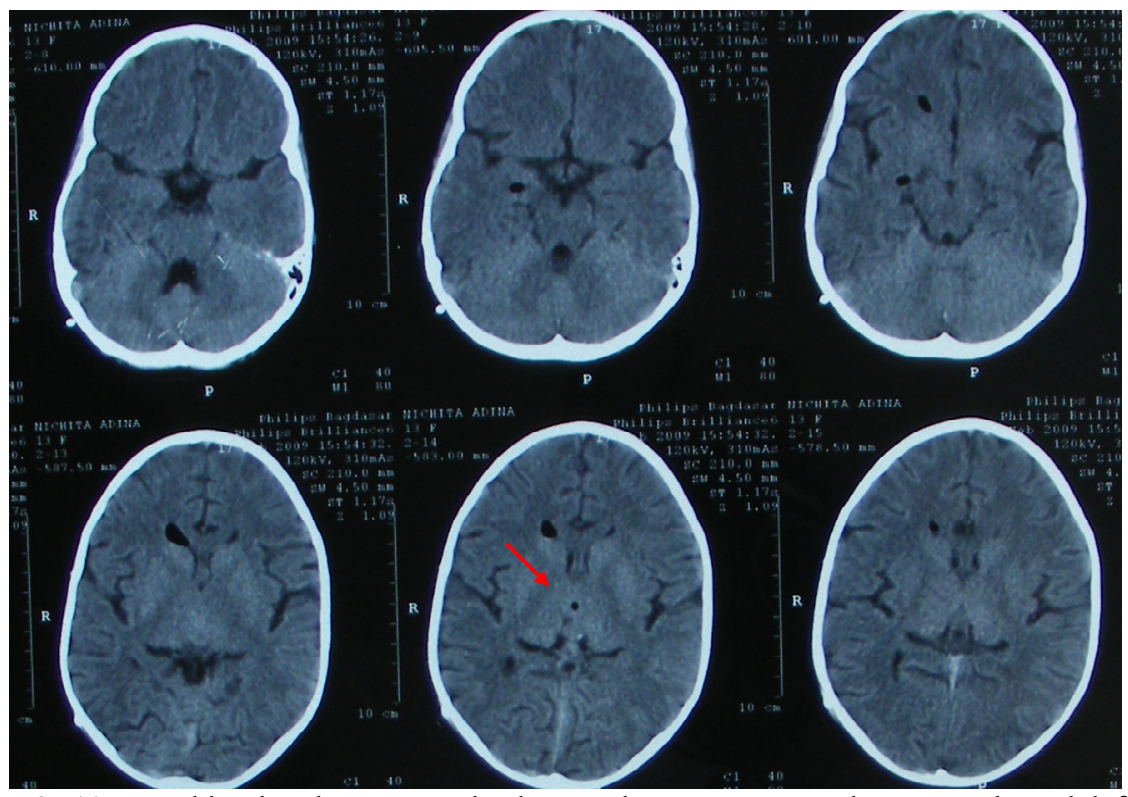

Figure 311 year-old girl with posterior third ventricle tumor mass without neurological deficits Postoperative unenhanced CT scan; Histopathological result: grade II astrocytoma
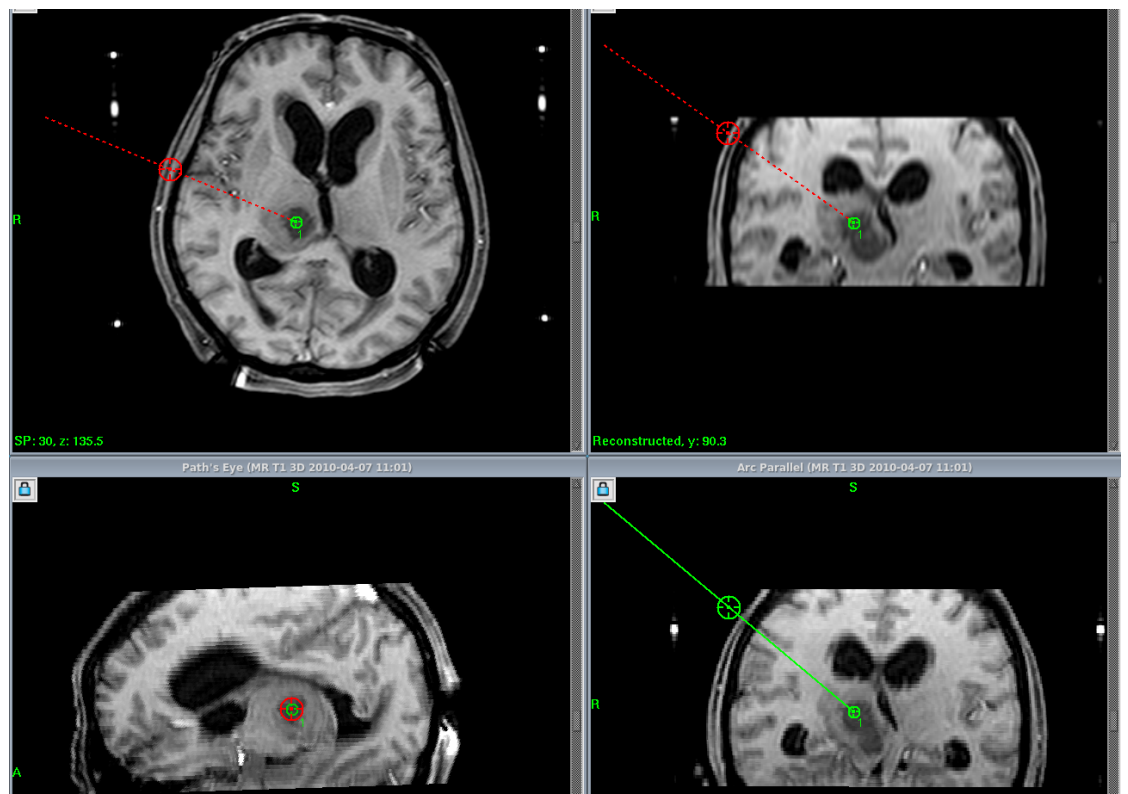

Figure 449 year-old female with right thalamic tumor mass - MR planning images 


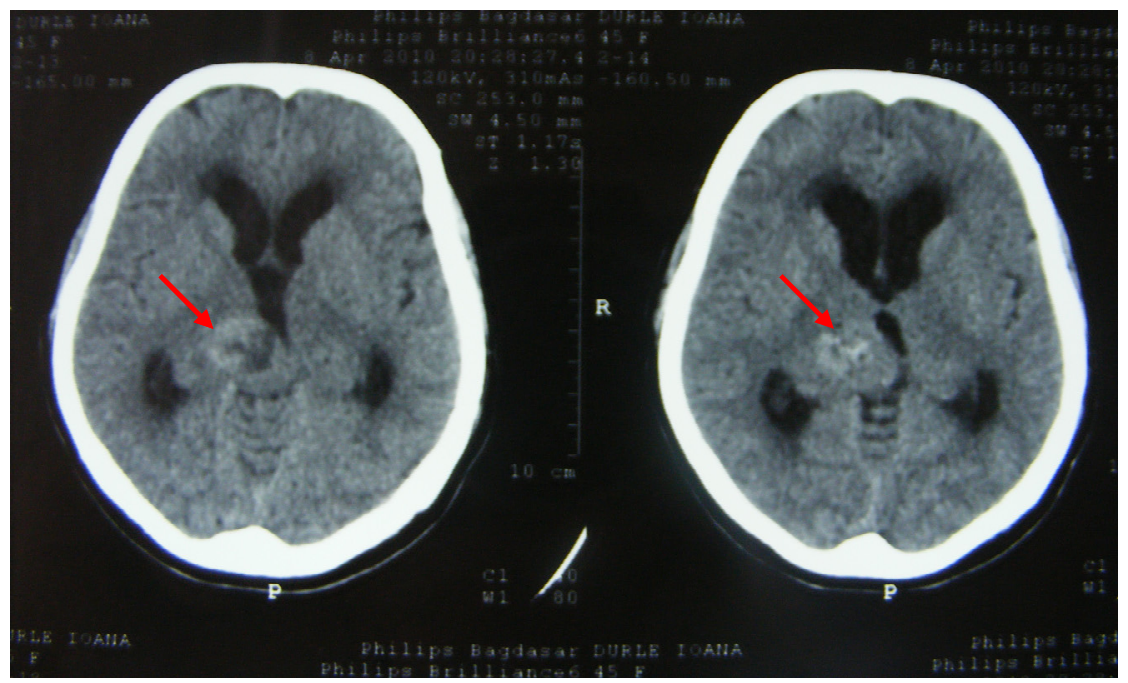

Figure 549 year-old female with right thalamic tumor mass - Postoperative unenhanced CT scan. Histopathological result: glioblastoma

In this series, the early postoperative (within 7 days after biopsy) mortality was 0 . One case of death $(1,2 \%)$ occurred at 10 days after biopsy. This was a case of infiltrating glioblastoma, located in right basal ganglia and insula, with mass effect and poor neurological status (somnolence and left hemiparesis), for whom the caregivers refused open surgery. At four days after biopsy, the general and neurological status of the patient suddenly deteriorated. CT scan showed an increased peritumoral edema and no sign of hemorrhage. Despite of the high doses of cortisone and mannitol administrated, the final outcome was unfavorable. There was no case of clinically significant hemorrhages after biopsy procedures. In 9 patients $(10,6 \%)$, CT scan showed evidence of minor hemorrhage at the biopsy site, which did not influence the neurological outcome of the patients. Temporary increasing of neurological deficits has been noticed in 7 patients $(8,2 \%)$. The incidence of immediate postoperative seizures was $7,1 \%$
( 2 cases of generalized and 4 cases of partial motor seizures). In three cases $(3,5 \%)$ there were signs of local infection which required intravenous antibiotics administration for 7 days. The median hospitalization time of the patients was 3,5 days.

\section{Discussions}

Stereotactic biopsy is a minimally invasive diagnostic procedure that takes small tumor samples with minimal risk for patients. The first use of a human stereotactic apparatus is credited to Ernest A. Spiegel and Henry T. Wycis, in 1947 (15). The field of stereotactic surgery has constantly developed since this first important step. The major achievement in combining the stereotactic frames and CT or MR scans, involved the development of a fiducial system (1). The availability of image-based stereotactic surgery opened new perspectives for the management of brain tumors. The most common stereotactic procedure became biopsy (7) and among the expansive intracranial 
DOI: 10.2478/v10282-012-0005-2

masses, the gliomas were the most suitable lesions for this approach, because many of them are infiltrative, or multicentric.

In this paper, the authors present their experience in 85 cases of gliomas with infiltrative or multicentric growth pattern, or located in eloquent area of the brain; all these features precluded the open surgical approach of the lesions. Therefore, the stereotactic biopsy remained the only options for these patients. For all procedures we used the Leksell stereotactic system which, in our opinion, is one of the most precise and easy to handle system due to its simple arc-centered principle. The popularity of Leksell stereotactic system is reflected by literature data. Therefore, the most important stereotactic apparatus are the Cosman-Roberts-Wells in USA, and the Leksell and Riechert- Mundinger systems in Europe $(6,7)$.

In our series, there was no major hemorrhagic accident. The explanation is that in the majority of cases we employed the high resolution MR images for planning the stereotactic procedure. The combination of gadolinium-enhanced T1weighted, $1 \mathrm{~mm}$ thickness slices and T2weighted, $2 \mathrm{~mm}$ thickness slices, enabled us to accurately visualize even the small blood vessels within the tumor centre or tumor periphery. The versatility of the stereotactic software, which includes the "path view" option, reveals to neurosurgeon all the structures (including the blood vessels) encountered along the trajectory path from the entry point to the target point. The importance of MR images in increasing the safety of stereotactic biopsy is underlined by the literature data. Therefore, the CTguided biopsy series have a relatively high incidence of major hemorrhagic complications, up to $5,6 \%(11,12,14)$. On the other hand, the MR-guided biopsy series have a significant lower frequency of major hemorrhagic complications to as low as approximately $0.7 \%(13,19)$. A similar correlation, but referred to the frequency of minor hemorrhage events, was observed by the authors of this paper. Between November 2008 and February 2011, we employed either MR or CT for stereotactic planning. However, the incidence of minor hemorrhages, revealed by the postoperative CT scan, strongly correlated with the type of neuroimaging tools used for planning procedure (CT scan with a higher incidence versus $\mathrm{MR}$ with a lower incidence). As a result of these findings, since March 2011, MR images were included in planning protocol for all cases. Therefore, the authors of this paper emphasize the importance of MR images (gadolinium-enhanced T1-weighted and T2-weighted) for stereotactic planning in order to avoid the hemorrhagic complications which may occur during the biopsy procedure.

The immunohistochemistry technique is a valuable diagnostic tool, which assess a precise diagnosis, including the malignity grade of glioma by evaluating the expression of Ki-67 marker, even in the presence of a very small amount of tissue sample. In our study, the immunohistochemistry technique was employed in approximately $25 \%$ of cases, especially when a small amount of tissue was available for histopathological analyses. If the molecular analysis of tumor samples were employed, the stereotactic biopsy could assess not only the diagnosis, but also could evaluate the prognosis and the response rate to chemotherapy. The relations between certain molecular markers and genetic mutations and the 
predictability of response to chemotherapy have been previously demonstrated. Thus, it is known that in the case of oligodendrogliomas, the presence of the $1 \mathrm{p} / 19 \mathrm{q}$ deletion is a favorable prognostic to chemotherapy $(4,9)$. The stereotactic group from Munich (F.W. Kreth) demonstrated the value of stereotactic biopsy procedure in assessing the molecular profiling of infiltrative low-grade gliomas (for TP53 mutation and loss of heterozygosity of $1 \mathrm{p} / 19 \mathrm{q}$ ) and glioblastoma (for MGMT methylation) with important implications in the prognosis and therapeutic management of the patients $(10,17,18)$. Therefore, one of the main priorities of our stereotactic group is to develop the molecular analysis of tumor samples in order not only to obtain an accurate diagnosis, but also to improve the therapeutic management of the patients.

\section{Conclusions}

The image-guided stereotactic biopsy represents now a safe and accurate diagnostic method for cerebral gliomas, which can favorably influence the therapeutic management of the patients. Using the MR images for planning improves the safety of stereotactic procedure by decreasing the incidence of hemorrhagic complications which may occur during biopsy.

\section{Correspondence author: F. M. Brehar \\ Emergency Clinical Hospital "Bagdasar- \\ Arseni”, Berceni Street 10-12, 041915 \\ Bucharest, Romania \\ e-mail:felixbrehar@yahoo.com}

\section{References}

1. Brown RA, Roberts TS, Osborn AG. Stereotaxic frame and computer software for CT-directed neurosurgical localization. Invest Radiol 1980;15:308-12
2. Buckner, J. C., O'Fallon, J. R., Dinapoli, R. P., Schomberg, P. J., Farr, G., Schaefer, P., Giannini, C., Scheithauer, B. W., and Ballman, K.V. (2007). Prognosis in patients with anaplastic oligoastrocytoma is associated with histologic grade. J. Neurooncol. 84, 279286.

3. CBTRUS (2005). Statistical Report: Primary Brain Tumors in the United States, 1998-2002. Published by the Central Brain Tumor Registry of the United States 4. Fabio M. Iwamoto, Linda Nicolardi, Alexis Demopoulos, Violetta Barbashina, Paulo Salazar, Marc Rosenblum and Adília Hormigo, Clinical relevance of $1 p$ and $19 q$ deletion for patients with WHO grade 2 and 3 gliomas, Journal of Neuro-Oncology , Volume 88, Number 3, 293-298, 2008

5. Giese, A. and Westphal, M. (1996). Glioma invasion in the central nervous system. Neurosurgery 39, 235250; discussion 250-252.22.

6. Gildenberg PL. Survey of stereotactic and functional neurosurgery in the United States and Canada. Appl Neurophysiol 1975;38:31-7.; 63

7. Gildenberg PL, Franklin P. Survey of CT-guided stereotactic surgery. Appl Neurophysiol 1985; 48:47780.

8. Greenberg Mark S, Handbook of Neurosurgery, Seventh Edition, N.Y., Thieme, 2010

9. Gregory Cairncross, Brian Berkey, Edward Shaw, Robert Jenkins, Bernd Scheithauer, David Brachman, Jan Buckner, Karen Fink, Luis Souhami, Normand Laperierre, Minesh Mehta, and Walter Curran, Phase III trial of chemotherapy plus radiotherapy compared with radiotherapy alone for pure and mixed anaplastic oligodendroglioma: intergroup radiation therapy oncology group Trial 9402, J Clin Oncol 24:2707-2714, 2006;10.

10. Kreth S, Heyn J, Grau S, Kretzschmar HA, Egensperger R, Kreth FW., Identification of valid endogenous control genes for determining gene expression in human glioma, Neuro Oncol. 2010 Jun;12(6):570-9. Epub 2010 Feb 5

11. Levine AB, Experience in the first 100 patients undergoing computerized tomography-guided stereotactic procedures utilizing the Brown-RobertsWells guidance system. Appl Neurophysiol 48: 45-49, 1985

12. Masamit Nishihara, Takashi Sasayama, Hiroshi Kudo, Eiji Kohmura, Morbidity of Stereotactic Biopsy for Intracranial Lesions, Kobe J. Med. Sci., Vol. 56, No. 4, pp. E148-E153, 2010

13. McGirt MJ, Villavicencio AT, Bulsara KR, Friedman AH, MRI-guided stereotactic biopsy in the diagnosis of glioma: comparison of biopsy and surgical resection specimen, Surg Neurol. 2003 Apr;59(4):27781; discussion 281-2

14. Mundinger F: CT stereotactic biopsy for optimizing 
DOI: $10.2478 / \mathrm{v} 10282-012-0005-2$

the therapy of intracranial processes, Acta Neurochir Suppl (Wien) 35: 70-74, 1985.

15. Spiegel EA, Wycis HT, Marks M, Lee AS. Stereotaxic apparatus for operations on the human brain. Science 1947;106:349-50

16. Stupp. R., Hegi, M. E., Gilbert, M. R., and Chakravarti, A. (2007). Chemoradiotherapy in malignant glioma: standard of care and future directions. J. Clin. Oncol. 25, 4127-4136.

17. Thon N, Eigenbrod S, Grasbon-Frodl EM, Ruiter M, Mehrkens JH, Kreth S, Tonn JC, Kretzschmar HA Kreth FW, Novel molecular stereotactic biopsy procedures reveal intratumoral homogeneity of loss of heterozygosity of $1 \mathrm{p} / 19 \mathrm{q}$ and TP53 mutations in World
Health Organization grade II gliomas, J Neuropathol Exp Neurol. 2009 Nov;68(11):1219-28; 12

18. Thon N, Eigenbrod S, Grasbon-Frodl EM, Lutz J, Kreth S, Popperl G, Belka C, Kretzschmar HA, Tonn JC, Kreth FW, Predominant influence of MGMT methylation in non-resectable glioblastoma after radiotherapy plus temozolomide, J Neurol Neurosurg Psychiatry. 2010 Sep 22; 13

19. Walter A. Hall, The safety and efficacy of stereotactic biopsy for intracranial lesions, Cancer, Volume 82, Issue 9, pages 1749-1755, 1 May 1998

20. Zulch K.J., Brain tumors. Their biology and pathology. Berlin: Springer-Verlag; 1986. 